\title{
sciendo
}

\section{A REVIEW ON BOVINE MASTITIS WITH SPECIAL FOCUS ON CD4 AS A POTENTIAL CANDIDATE GENE FOR MASTITIS RESISTANCE - A REVIEW*}

\author{
Arsalan Rasheed ${ }^{1,2}$, Tahir Usman ${ }^{1 \bullet}$, Kamal Niaz $^{3}$ \\ ${ }^{1}$ College of Veterinary Sciences and Animal Husbandry, Abdul Wali Khan University, Mardan 23200, \\ Pakistan \\ ${ }^{2}$ Department of Zoology, Faculty of Chemical and Life Sciences, Abdul Wali Khan University, \\ Mardan 23200, Pakistan \\ ${ }^{3}$ Department of Pharmacology and Toxicology, Faculty of Bio-Sciences, \\ Cholistan University of Veterinary and Animal Sciences, Bahawalpur-63100, Pakistan \\ •Corresponding author: tahircau@gmail.com, tahirusman@awkum.edu.pk
}

\begin{abstract}
Mastitis is an inflammation of the mammary gland, caused by the invasion and duplication of Escherichia coli (E. coli), Staphylococcus uberis (S. uberis) and Staphylococcus aureus (S. aureus) and a wide variety of other microorganisms through teat or damaged nipple, decreasing potential milk production in the affected quarter of mammary gland. Economic, animal productivity, international trade and animal welfare issues associated with mastitis play an important role in the agricultural industry. Therefore, worldwide dairy cattle breeding programs are trying to breed cows with improved resistance to mastitis. Mastitis cannot be eliminated but can be reduced to a low level. This can be achieved by breeding strategies, reducing the exposure to pathogen and increasing the resistance to intramammary infection. Numerous therapeutic, prophylactic and management techniques are used to control and reduce the mastitis. However, a widely proposed strategy is marker assisted selection using candidate gene approach which is based on improving the host genetics. One of them is cluster of differentiation 4 (CD4) gene, which is a glycoprotein located on receptors of immune cells. CD4 exhibits an essential role in a variety of inflammation related conditions in many species. Therefore, $C D 4$ as a candidate gene for resistance to mastitis has received considerable attention. The review is based on a study of CD4 in association with improving resistance to mastitis and it may be helpful in formulating breeding programmes and marker assisted selection to lower the mastitis.
\end{abstract}

Key words: mastitis, inflammation, candidate gene, $C D 4$, mammary gland

\footnotetext{
*This research was financially supported by the SRGP-536, HEC Pakistan.
} 


\section{Background of the research}

The word mastitis is derived from two Greek words i.e. mastos which means "breast" and itis which means "inflammation". Therefore, mastitis refers to inflammation of the mammary gland (Campbell and Marshall, 2016). The mammary gland tissue is protected by two defence systems of the immunity i.e. innate (nonspecific) immunity and the acquired (specific) immunity. Both innate and acquired immunity interact to provide protection against mastitis causing microorganisms (Burvenich et al., 2003).

Mastitis is classified into two types i.e. clinical mastitis (CM) and sub-clinical mastitis (SCM). CM may be mild or severe. Mild CM may show discoloration of the milk color, shape and smell with flakes or clots in the milk while the animal having severe CM shows clear symptoms of disease such as heat, swelling of udder, abnormal secretions, fever and loss of appetite. SCM is the most prevalent form of mastitis in which there is absence of evident signs. SCM could develop into a clinical disease if not detected properly using somatic cells count (SCC) values (Sharma et al., 2011). Mastitis is further classified as acute and chronic depending on severity and duration of infection. Acute mastitis is characterized by a sudden onset whereas chronic mastitis is characterized by an inflammatory process that lasts for months.

\section{Mastitis: A global disease of dairy cattle}

Mastitis is one of the most common diseases compromising dairy animals' health and welfare worldwide (Campbell and Marshall, 2016). Milk production is badly affected by linking milk production with poor milk quality, thus causing huge economic loss to the milk industry all over the world (Hogeveen et al., 2011; Halasa et al., 2007). Reducing mastitis and SCC in milk are both public health safety and food quality initiatives. Over time, the use of antimicrobial agents or drugs has increased the number of antimicrobial resistant microbes globally, and the use of any of these agents results in the development of resistant strains. Improper use such as the wrong dose, drug or duration can contribute most to increased anti-microbial resistance without improving treatment outcome. The increase in resistance to antibacterial agents has raised serious concerns worldwide from both public health and food safety perspectives, putting their use in food producing animals under constant scrutiny over the years. Resistance against mastitis in cattle is attracting the attention of modern livestock for breeds of cows with improved resistance towards mastitis.

Despite many years of research, it remains the most economically damaging and zoonotic potential disease for dairy industry and consumers worldwide, irrespective of species of animal (Ojo et al., 2009). During last several decades, it has become as expensive disease affecting the dairy industry and consumers globally (Fourichon et al., 2000). Researchers have found that there is high risk of developing SCM in period of early lactation (Viegher et al., 2005). The disease is of great importance in the agricultural industry due to problems associated with the reduction of output, public health, economy, international trade, animal productivity and welfare (Hogeveen et al., 2011; Coulon et al., 2002). The purpose of various studies is to reduce the mastitis and obtain high quality milk by monitoring SCM. 
Consequently, the aim of this review is to provide a detailed overview of mastitis, updating the literature on mastitis, to determine the role of $C D 4$ in inflammatory conditions of different species. CD4 is known to play an exceptional role in immune mechanisms against a variety of inflammatory diseases in many species, most importantly in the immune response (developing resistance) during the development of mastitis in dairy animals. The current review focuses on $C D 4$ as a potential candidate gene for resistance against mastitis to open new doors for improving milk production and cow udder health.

\section{Causative agents of mastitis}

More than one hundred different microorganisms including bacteria, viruses, mycoplasma, yeast and algae have been isolated from bovine mastitis. The most frequently isolated among them are staphylococci, streptococci and gram negative bacteria (Oliver et al., 2004). $70-80 \%$ of all the CM and SCM cases were either infected with Staphylococcus aureus or Streptococcus agalactiae in cows and buffaloes (Memon et al., 1999). Some studies show that Streptococcus dysgalactiae, Klebsiella pneumoniae, Streptococcus bovis, Pseudomonas aeruginosa, Nocardia, Prototheca wickerhamii, Brucella abortus, Trueperella pyogenes, Prototheca zopfi, species of Pasteurella, Enterococcus, Corynebacterium, Arcanobacterium and Mycoplasma, and yeast are also involved in mastitis progression (Dieser et al., 2014; Muhasin et al., 2014).

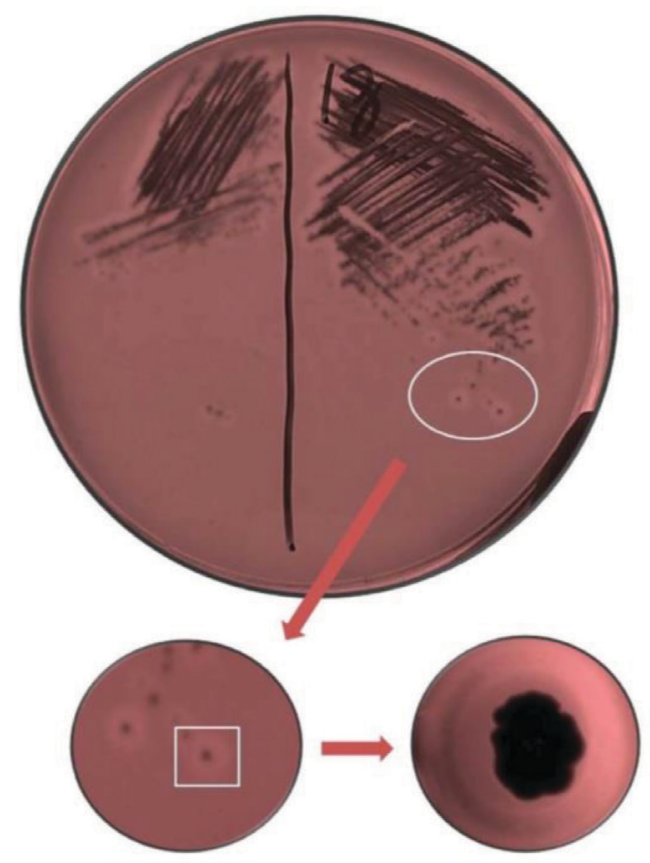

Figure 1. Culture and the identification of Staphylococcus aureus in Baird-Parker agar. A single colony is amplified (Source: Song et al., 2016) 


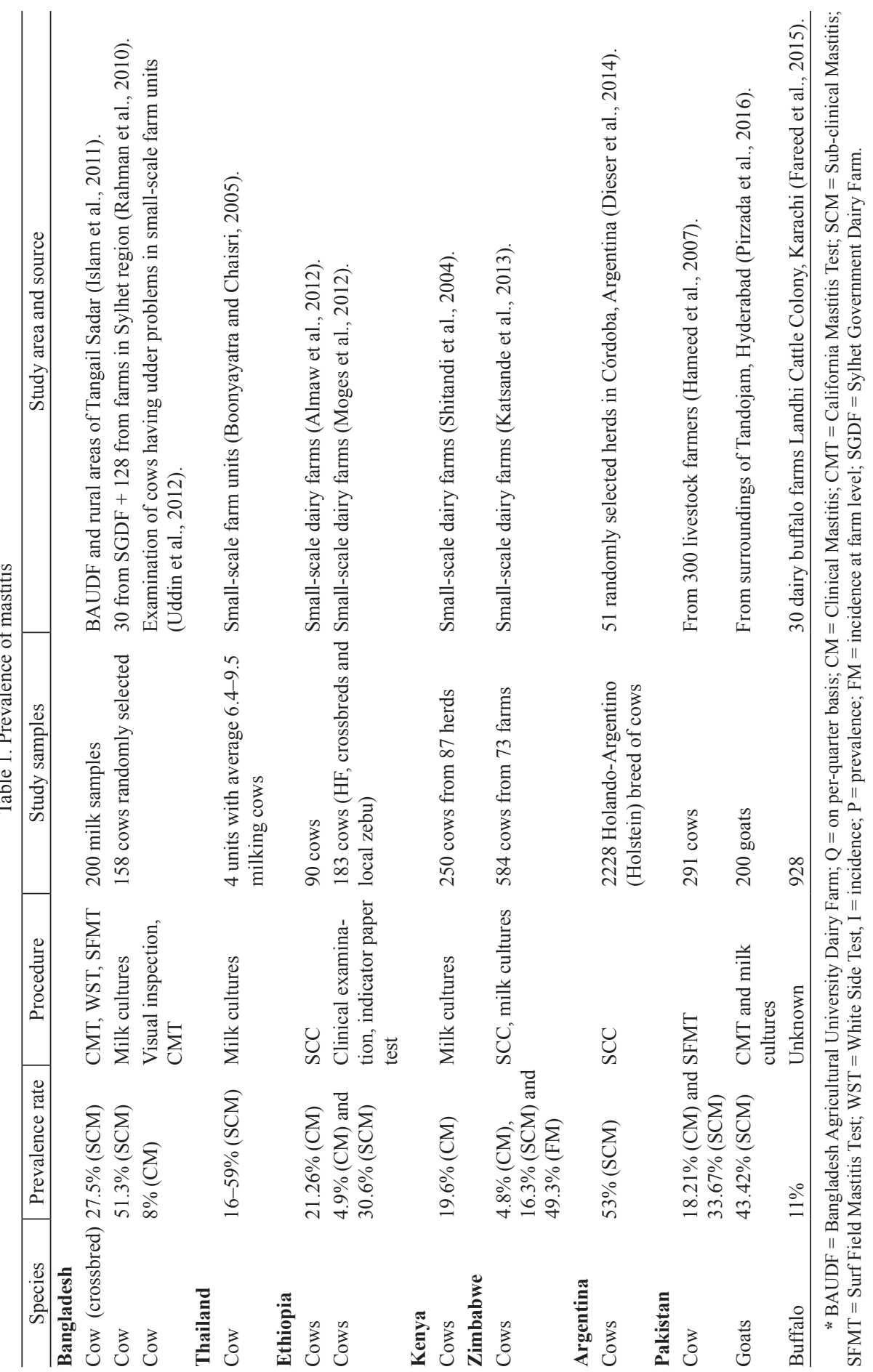




\section{Prevalence rate of mastitis}

It is difficult to determine the prevalence rate of mastitis but the estimated figure is about 35-50 cases/100 cows annually (Bradley et al., 2001). Mastitis is on the top of list of diseases in dairy cattle in 2013-14 according to The Swedish Board of Agriculture (http://www.jordbruksverket.se/). Previous studies conducted in different countries reported different prevalence rates of mastitis in dairy cattle indicating the distribution and economic importance of mastitis such as Nam et al. (2010) from Korea; Boonyayatra and Chaisri (2005) from Thailand; Ameh and Tari (2000) from Nigeria; Iraguha et al. (2017) from Rwanda; Ndegwa et al. (2000) from Kenya; Gebreyohannes et al. (2010) and Megersa et al. (2010) from different parts of the Ethiopia; and Kozacinski et al. (2002) from Croatia.

Prevalence rate of mastitis in Asia is increasing with the development of high milk producing breeds of buffaloes and cows. Studies carried out in Kyber Pakhtunkhwa (KP) Province (Sammiullah et al., 2000), and Punjab such as Muzaffar Garh (Bachaya et al., 2011), and Faisalabad (Bilal et al., 2004) etc. show that mastitis is the most frequent problem identified by the small-scale farmers of Pakistan. Various studies conducted in different countries are shortened below (Table 1).

\section{Factors contributing to mastitis}

Many factors affect the incidence of mastitis, such as the productive stages of the cow, the number of lactations, the management of the herd, the temperature of the environment, the humidity, the weather, the breeds and the characteristics of the lactation. Table 2 shows the various factors and management mechanisms that produce variation in somatic cells in the dairy herd.

Table 2. Factors increasing and decreasing milk SCC

\begin{tabular}{ll}
\hline \multicolumn{1}{c}{ Factors Increasing Milk SCC } & \multicolumn{1}{c}{ Factors Decreasing Milk SCC } \\
\hline Udder infection or injury & Healthy udder \\
Change in housing and feeding & Post milking teat dipping \\
Increase in parity & Hygienic milking practices \\
Cows calving during warm and rainy weather & $\begin{array}{l}\text { Hygiene, vaccination, nutrition, and housing } \\
\text { management practices }\end{array}$ \\
Increase in stage of lactation & Clean animal surroundings \\
Unhygienic/Incomplete milking & Regular udder screening \\
Unpasteurized milk and milk products & Pasteurization \\
Unhygienic cow surroundings & Feeding antioxidants \\
Vitamin E and selenium deficiency & Rationalize feeding diets \\
Hot humid climates & Treating infected cows \\
Keeping sick cows with healthy cows & Awareness about mastitis \\
Any other stress & Selection against mastitis \\
\hline
\end{tabular}

\section{Human health risks associated with mastitic milk}

Milk drawn from an infected animal is the main source of pathogens. Some bacterial toxin produced in the milk cannot be destroyed by pasteurization techniques (National Mastitis Council, 1996). Such milk is dangerous for public health as it can transmit pathogenic bacteria to humans through the food chain. Milk is discarded by 
the producer in severe $\mathrm{CM}$ due to visible abnormalities and would not enter the food chain. However, milk of cows in SCM having no visible changes is mixed into bulk milk and thus enters the food chain and can be dangerous to humans (Hameed et al., 2007). Milk contaminated with bacteria from affected cows is unsuitable for human consumption and may cause food poisoning or in rare cases provides mechanism of spread of disease in humans. It was discovered earlier that milk can transmit tuberculosis, brucellosis, diphtheria, scarlet and Q-fever to humans. However, they can be controlled through pasteurization but various bacteria still contribute to illness and disease outbreaks (Gilmour and Harvey, 1990).

\section{Economic losses caused by mastitis}

Mastitis is an expensive disease in the dairy industry economically. It is estimated that mastitis costs over $\$ 1.8$ billion per year in the USA alone affecting $1 / 3$ of all dairy cows (Schroeder, 2012). Each incidence of CM costs about $\$ 444$ in large US dairy herd during the first 30 days of lactation (Rollin et al., 2015). An estimate shows a cost of about €97 per cow annually due to CM in Sweden (HagnestamNielsen and Ostergaard, 2009). Economic losses caused by mastitis, either CM or SCM can be brought down to a few categories i.e. effects on milk composition and quality, milk yield losses, lethality and occurrence of other diseases like brucellosis, lymphadenitis, leptospirosis, listeriosis, melioidosis, Q-fever, staphylococcal food poisoning, toxoplasmosis and tuberculosis, treatment costs i.e. veterinarian fees and cost of drugs, labour costs, premature culling and replacement etc.

\section{Pathogenicity of mastitis}

Mastitis pathogens can be divided into contagious and environmental. The primary reservoir of contagious pathogens is an infected udder whereas a contaminated environment is the primary reservoir of pathogens causing environmental mastitis. Mastitis is a complex and multifactorial disease in which pathogens enter the udder and cause an infection. However, for pathogens to enter the mammary glands and establish themselves to the point that they cause an infection, a multitude of other factors may be involved: hygiene, housing, climate, milking machines, feeding and genetic factors (Karima, 2013), as the occurrence of disease is an outcome of interplay between three major factors: infectious agents, host resistance, and environmental factors (Gera and Guha, 2011). The factors might be an individual genotype, the pathogens involved, the environment of cattle, milking techniques and interaction among them as shown in Figure 2.

When pathogens attach to epithelial cells, they enter and evade the host defense mechanism and penetrate into the deeper regions (Almeida et al., 1998). Mastitis pathogens overcome defense mechanism at mucosal membranes by invading the mammary epithelial cells and adhere to mammary mucosal membranes causing an infection of mammary tissues. When the pathogens gain entry into the udder, they find nutrients and multiply rapidly. Cow's udder is a primary reservoir of contagious mastitis pathogens. These pathogens commonly transmit among cows by contact with infected milk. Their metabolic by-products cause poisoning of the udder tissues resulting in an inflammation. The inflammation is due to the infiltration of leukocytes 
i.e. cow's autoimmune response to the toxic metabolites released by the bacteria. These toxic metabolites can damage the milk secreting tissues and ducts throughout the udder. Observations likely reflect the unsuccessful combat of mammary glands against the invading bacterium and that the sensitivity or responsiveness of mammary glands to inflammatory signals decreased as infection progressed. In experimentally induced ovine intramammary inflammation by $S$. epidermidis, counts of leukocyte subsets (including $C D 4, C D 8, W C 1$, and $M H C I I$ ) temporarily decreased and then subsequently increased, while the expression of some adhesion molecules ( $C D 11 \mathrm{~b}$ and $C D 18$ ) on polymorphic nuclear neutrophils decreased after $24 \mathrm{~h}$ (Winter and Colditz, 2002).

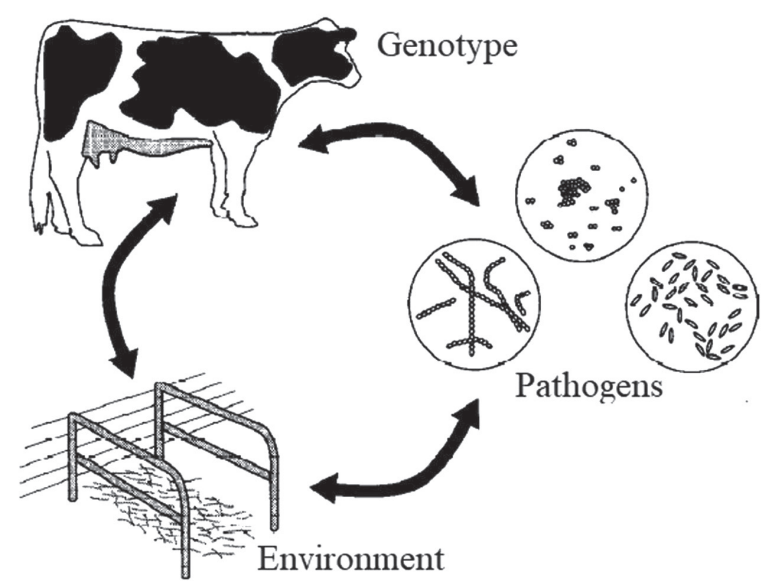

Figure 2. Pathogenicity of mastitis (Source: Schroeder, 2012; Oviedo-Boyso et al., 2007)

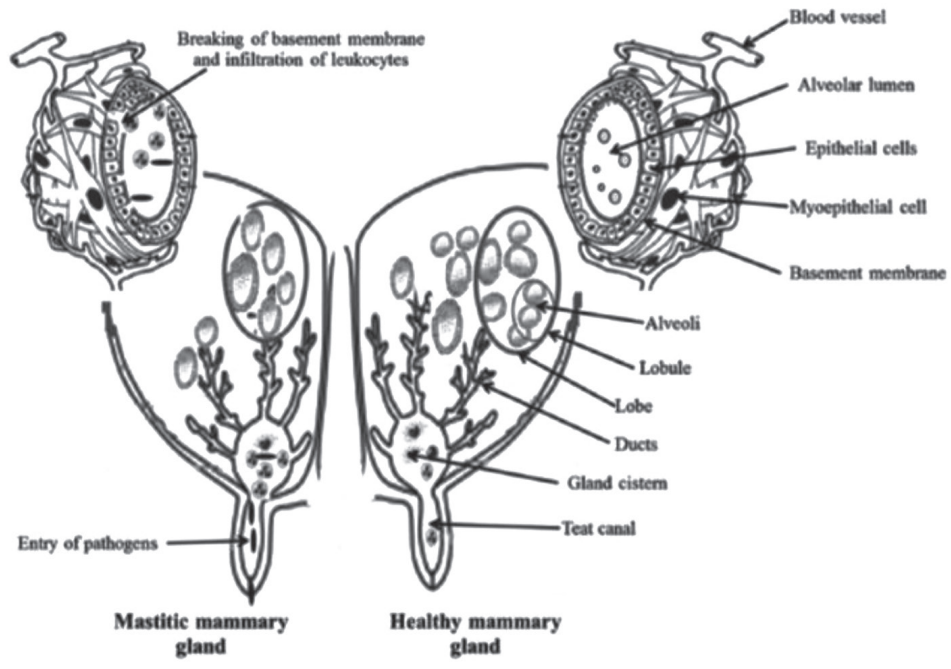

Figure 3. Mastitis and healthy mammary gland (The penetration of pathogens to the internal tissues of mammary gland incites the trafficking of various immune cells to the site of inflammation as a result elevation of SCC occurs in the secreted milk. Source: Alhussien and Dang, 2018) 


\section{Diagnosis}

It is important to diagnose, monitor and predict disease by several methods including the California Mastitis Test (CMT), Surf Field Mastitis Test (SFMT), the Modified White Side Test, $\mathrm{SCC}, \mathrm{pH}$, and catalase tests etc. These tests are preferred to be done screening milking animal for SCM as they can be used easily, yielding rapid as well as satisfying results (Joshi and Gokhale, 2006).

\section{California Mastitis Test (CMT) and pH test}

A small sample of milk (approximately $1 / 2$ teaspoon) is collected from each quarter into a plastic paddle with 4 shallow cups marked as A, B, C and D. An equal amount of CMT reagent is added to the milk and the paddle is rotated for approximately 10 seconds to properly mix the materials. The score is read while continuing to rotate the paddle. Results are recorded and divided into four types which are moderate $(+)$, severe $(++)$, more severe $(+++)$ and very severe $(++++)$ based on the level of precipitation (coagulation) (Mellenberger, 2001).

The $\mathrm{pH}$ of normal milk is 6.6 to 6.7 while infected milk has higher $\mathrm{pH}$ to alkalinity side which is approximately $7.2 \pm 0.2$ equal to blood pH (Figure 3). Both SFMT and $\mathrm{pH}$ test are good and economic methods used for the diagnosis of mastitis.

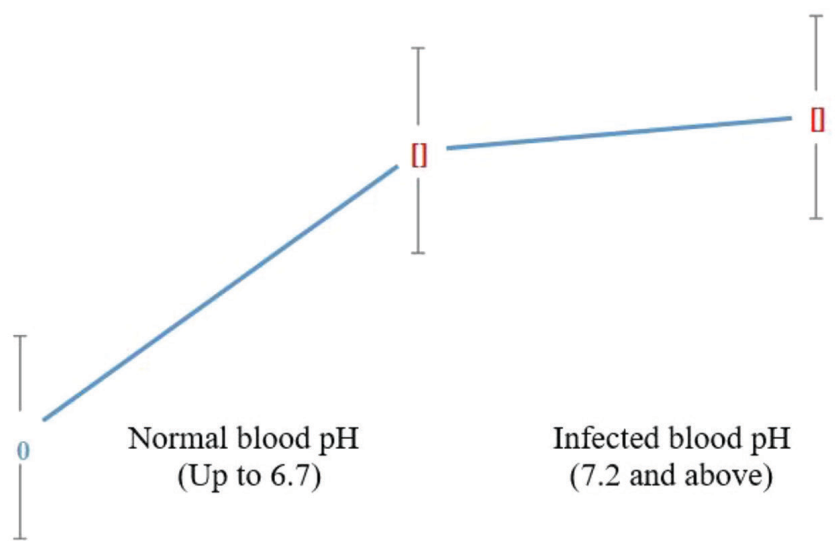

Figure 4. pH comparison of normal and infected cattle blood

\section{Somatic cell count (SCC)}

SCC is the number of somatic cells per milliliter of milk. Milk SCC is a useful indicator of udder health and diagnostic parameter for SCM. Therefore, it is widely used as an indirect index for mastitis control (Pant et al., 2007). White blood cells (leukocytes) constitute the majority of somatic cells. Somatic cells are normal components of milk. The number of somatic cells increases in response to pathogenic bacteria like Staphylococcus aureus, which is a causative agent of mastitis. Cow milk SCC of $>20,000$ cells $/ \mathrm{ml}$ indicates mastitis (International Dairy Federation, 1999). In healthy quarters of dairy cows, SCC per milliliter milk ranges from $2 \times 10^{4}$ to $2 \times 10^{5}$ cells $/ \mathrm{mL}$ milk while in an infected quarter, SCC ranges from $3 \times 10^{5}$ to $9 \times 10^{7}$ 
cells/mL milk (Holmes and Wilson, 1984; Kehrli and Schuster, 1994). Because of highly positive genetic correlation (0.71-0.97) between SCC/SCS and CM (Rupp and Boichard, 1999; Hinrichs et al., 2005; Sorensen et al., 2009), a worthy approach being used is to select cattle with genetically low SCC level to reduce the risk of mastitis in dairy cattle (Koivula et al., 2005; Swanson et al., 2009; Stear et al., 2001).

\section{Acute phase proteins in bovine milk}

Acute phase protein considered as a good tool for detecting the severity of inflammatory conditions in dairy cow (Brodzki et al., 2015). Milk amyloid A is an acute phase protein which increases in concentration with an inflammatory response in the udder. Due to less influence of other physiological factors, milk amyloid A may be a more sensitive marker than SCC. Low milk amyloid A is an indication of healthy quarter while an increased level $(<500 \mathrm{mg} / \mathrm{ml})$ of milk amyloid A indicates an inflammatory response (Glass et al., 2005).

\section{Approaches for increasing mastitis resistance}

Incidences of mastitis can be lowered by decreasing the exposure to pathogens or by increasing resistance of cow to intramammary infection (Smith et al., 2000). Resistance could be defined as the ability to avoid an infection of the udder or quick recovery from any infection. With the advancement of molecular techniques mapping and tagging of genes, identification of genetic markers, studying candidate genes and detection of quantitative trait loci (QTL) are being used to make genetic improvement in breeding stock (improve production and disease resistance traits) through marker assisted selection (Kolbehdari et al., 2009). Using these techniques may be useful tools in understanding the genetic determinism of mastitis resistance.

\section{Marker assisted selection}

Modern developments in biotechnology have made it possible to unravel bulky genetic polymorphisms at DNA level. Functional mutation is used in marker assisted selection (MAS) which is basically responsible for phenotypic variations (Dekkers and Hospital, 2002). Two main approaches are used to study markers (genes associated with mastitis resistance). One is to detect and localize the QTL while the other is candidate gene approach which looks for single nucleotide polymorphisms (SNPs) in the genes which are related to mastitis. The SNPs within the candidate genes are tested for better understanding their effects and association with quantitative traits. Another method to locate genomic variants or markers is genome wide association studies (GWAS). This method utilizes the information on genetic markers like SNPs to study their association with a trait of interest assuming that the genetic marker is in linkage disequilibrium with or close to a causative mutation (Goddard and Hayes, 2009). GWAS have been used to identify marker or SNP and their association with mastitis related traits and many QTLs in cattle.

Mastitis resistance is controlled by several loci, each of which is responsible for small amount of overall variation. Variety of SNPs exist across the genome of different species which are used in selection program over other genetic markers due to their relatively less frequent mutation rate, easy and cost effective genotyping 
(Hinds et al., 2005). These advancements in biotechnology result in a large number of alternative methods of genetic markers for studying the required traits and using them in MAS, including microsatellites, amplified fragment length polymorphisms (AFLPs) restriction fragment length polymorphisms (RFLPs) to detect SNPs and for identification of the important genes for genetic improvement. Various techniques are being used as control measures for bovine mastitis including candidate gene approach which focuses on improving the host genetics through MAS (Pant et al., 2007). However, utilization of MAS in dairy cattle produces additional genetic and economic gains and can utilize the candidate genes associated with health and other production traits (Ateya et al., 2016). In the present study identification of SNPs in inflammation related candidate gene, mRNA expression and DNA methylation level and their association with mastitis traits have been evaluated.

\section{Candidate gene approach}

Two main approaches are used to find loci of interest controlling phenotypic traits. One is candidate gene approach and the other is whole genome sequencing based on linkage mapping on DNA markers. A candidate gene can be defined as a gene with biological effects on the physiology of a trait of interest (functional) or as a gene closely linked to a functional gene (positional). This approach is considered as a very powerful method and is used to discover SNP even with a minor effect. Sometimes candidate gene provides information as a true causative gene. Studies using candidate gene approach for conducting genetic association focuses on associations of genetic variation within known genes of interest with phenotypes or disease states. This method is commonly used in association study due to its simplicity for obtaining samples and genotype data (Singer, 2009).

As described earlier, mastitis resistance is a complex function involving various biological pathways, molecules and cells. Therefore, many functional candidate genes could play an important role in the determinism of the function (Detilleux, 2002). During last decade the genes associated with the immune response have been investigated for the presence of SNPs and associations with mastitis related traits. Functionally, CD4 gene is tested for association to diseases like asthma, HIV infection, CD+ lymphocytic deficiency, breast neoplasms etc. The gene participates in pathways such as antigen dependent B cell activation, cytokines and inflammatory response etc. Hence, $C D 4$ has received some attention as a candidate gene for mastitis resistance.

\section{Cluster of differentiation 4}

CD4 gene was discovered in the late 1970s. CD4 was originally known as "leu3" and "T4" after the anti-human CD4 monoclonal antibody clone (OKT4) monoclonal antibody that reacted with it before being named as CD4 in 1984. CD4 was originally described as helper T lymphocyte antigen in 1979 by Ellis Reinherz. CD4 is present on chromosome 12 in humans, on chromosome 6 in mice while located on chromosome 5 in cattle. In humans, CD4 is highly expressed in thymus, liver and spleen while weakly in myeloid cells such as monocyte, dendritic cells and neutrophils (Bialecki et al., 2011). In humans, the gene contains 35 introns, 10 exons, 
a length of 31, 326 base pairs and encodes CD4 protein. CD4 protein a member of the Ig superfamily due to the presence of four extracellular Ig-like domains. It also contains a highly basic cytoplasmic tail of 38 amino acids containing three serine residues which can be phosphorylated and a hydrophobic trans-membrane domain. It mediates both recognition and adhesion functions due to the interaction of $\mathrm{N}$-terminal Ig-like domains with the non-polymorphic $\alpha 2$ and $\beta 2$ domains of MHC class II molecules. Cytoplasmic tail of CD4 has sites which facilitates physical association with LCK (lymphocyte-specific protein thyrosine kinase) (Mak and Saunders, 2006). LCK is critically important for T-cell development in the thymus. In mammals, CD4 is composed of four extracellular immunoglobulin (Ig)-like domains, a transmembrane region and a cytoplasmic tail (Kono and Korenaga, 2013; Horejsi, 2003). This cytoplasmic tail, which contains a conserved binding site, interacts with the LCK to induce the activation of T cells.

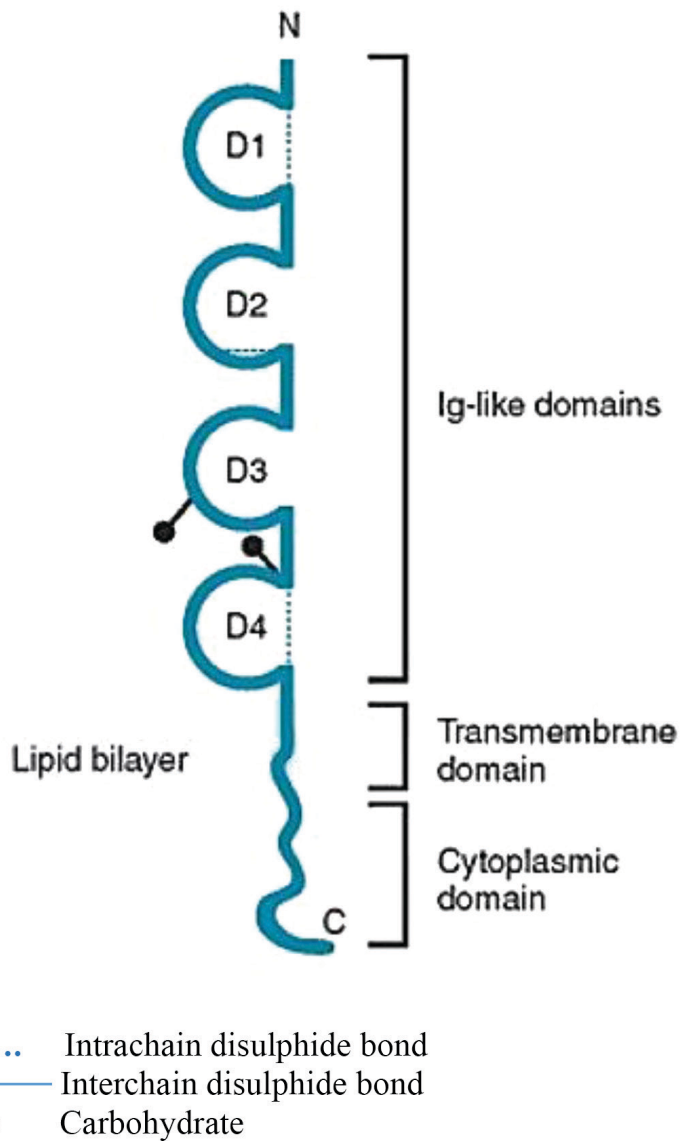

Figure 5. Structures of the CD4 co-receptors, Ig-like domains and glycosylation sites. (Source: Mak and Saunders, 2006; Horejsi, 2003) 
Genes encoding the bovine major histocompatibility complex (MHC or BoLA) molecules (BTA23) and playing an essential role in the regulation and induction of acquired immune response are studied extensively. The MHC is classified as class I, II and III genes containing clusters of genes. Class I molecules are expressed at the surface of all nucleated cells and interact with cytotoxic T lymphocytes (CD8+). Expression of class II molecules is restricted to the antigen presenting cells. They are involved in antigen presentation to helper T lymphocytes cells (CD4+) and in the development and classification of T cells (Rothschild et al., 2000). CD4 is a coreceptor in MHC class II-restricted T-cell activation, thus critical for the activation of the mature T cells. In T-cells it acts as a co-receptor for MHC class II molecule and forms peptide complex. Antigens presented by class I peptides are derived from cytosolic proteins and class II peptides are derived from extracellular proteins. CD4 interact simultaneously with T-cell receptor (TCR) and MHC class II presented by antigen presenting cells. In turn, it recruits the Src kinase LCK to the vicinity of the TCR-CD3 complex. LCK then initiates different intracellular signaling pathways by phosphorylating various substrates ultimately leading to lymphokine production, motility, adhesion and activation of T-helper cells. In other cells such as macrophages or NK cells, it plays a role in differentiation/activation, cytokine expression and cell migration in a TCR/LCK-independent pathway.

The control of CD4 gene expression is believed to be linked directly to the signaling events that mediate T-cell development and is directly dependent on the CD4 promoter. It also plays a role in the differentiation of thymocytes and the regulation of T-lymphocyte/B-lymphocyte adhesion. CD4 is expressed in a large proportion (80-90\%) of thymocytes and over $50 \%$ of the peripheral blood T cells (helper/inducer subtype). Most thymocytes co-express $C D 4$ and $C D 8$. Monocytes, macrophages and Langerhans cells express $C D 4$. The majority of post-thymus T-cell neoplasms are CD4+ (Faramarz, 2008). The expression of CD4 is detected only in immune tissues. White blood cell and CD4+ T helper cells are important part of the cattle immune system. They are frequently referred to asset-helper cells or T4 cells and CD4 cells. They are known as helper cells due to their main roles of sending signals to other types of immune cells, including CD8 killer cells which then destroy the infectious particle. If CD4 cells become depleted, for example in untreated infection, or following immune suppression prior to a transplant, the body is left vulnerable to a wide range of infections that it would otherwise have been able to fight against infection foreign body.

\section{Role of CD4 in inflammation in different organisms}

CD4 is a glycoprotein located on receptors of immune cells i.e. monocytes, T helper cells, macrophages, and dendritic cells. CD4 is known to play an essential role in a variety of inflammation related conditions in many species. CD4 and $L A G$ 3 both are powerful candidate genes in many inflammatory responses in different species (Wang et al., 2017; Miyagawa et al., 2016). Polymorphisms in CD4 gene have been reported in sheep (Boscariol et al., 2006) and in miniature swine (Gustafsson et al., 1993). The chicken CD4 gene has remained conserved in evolution (Koskinen et al., 2002). Oyugi et al. (2009) reported that promoter methylation of 
the CD4 gene was down-regulated in chickens after viral infection. CD4, CD8 and CD21 antigens on lymphocytes and IL-10 concentration in the peripheral blood of young dogs suffering from atopic dermatitis complicated by purulent inflammation of the skin. An association between polymorphisms of the CD4 gene and T lymphocyte subpopulations in pigs and their effects on the CD4 gene expression level in immune tissues was found by Xu et al. (2013). The silencer (S4) is a 434 bp core element in the first intron of CD4 that was identified by its ability to inhibit expression from reporter constructs in cell lines and in both double-negative (DN) thymocytes and CD8+ T cells of transgenic mice. Deletion of this element in the germ line of mice caused CD4 depression in DN thymocytes and CD8+ cytotoxic T cells, confirming its physiological function (Leung et al., 2001; Zou et al., 2001). Zhao and Lacasse (2018) speculated that increased methylation frequency of CpG_2 may lead to the decreased expression of $C D 4$, which may have some kind of influence on $\mathrm{T}$ lymphocyte subpopulation traits and the immunity of population. The $C D 4$ gene can be taken as a vital candidate gene on account of its important function in porcine immunity (Xu et al., 2013).

\section{Role of $C D 4$ in other diseases}

A study reported that cows infected with Staphylococcus aureus had lower proportions of $C D 4+\mathrm{T}$ cells in peripheral blood if compared with uninfected cows (Rivas et al., 2007). A recent study suggested that CD4 could be used as a candidate gene for milk production traits and the identified SNPs as potential genetic markers to improve fat percentage in Chinese Holstein cattle (Usman et al., 2016). CD4 gene promoter contains $\mathrm{CpG}$ island and it was reported that promoter methylation of the CD4 gene was down-regulated in L72 chickens only after Marek's disease virus infection (Oyugi et al., 2009). Usman et al. (2016) found 10 SNPs in CD4 having significant association with fat percentage in cattle. CD4 acts as a receptor for human herpes virus 7 (HHV-7) (PubMed: 7909607). Mutagenesis and monoclonal antibody studies have shown that the region spanning amino acids 40-60 of the D1 domain of CD4 is the part of the molecule directly in contact with the viral gp120 for all isolates examined, with residues Phe43 and Arg59 being particularly important. In response to microbial infection, $C D 4$ participates in the development of T-helper cells in the thymus and triggers the differentiation of monocytes into functional mature macrophages. Loss of memory CD4+ T cells impairs replenishment of the mature T cell pool, thus contributing to systemic CD4+ T cell depletion (Mattapallil et al., 2005).

CD4 regulatory $\mathrm{T}$ cells (Tregs) normally comprise a small subset (5 to 6\%) of the overall CD4 T cell population. Naturally occurring CD4 CD25 Tregs as well as other CD4 Tregs include Tr-1 cells and T helper 3 (Th3) cells secreting IFN- $\gamma$ and IL-10, and high levels of TGF- $\beta$, IL-4 and IL-10 respectively. Mukherji et al. (1989) observed the existence of CD4 T cell mediated immune suppression in animal tumor models and human cancer. Although, due to lacking reliable molecular markers such cells are not well characterized. An elevated proportion of CD4 CD25 Tregs in the total CD4 $\mathrm{T}$ cell populations is a common occurrence in a variety of human cancers, including lung and breast cancers as well as ovarian tumors and human melanoma (Wang et al., 2006). Other than this feature, which distinguishes them from CD4+ 
Tregs, very little is known about how CD8 Tregs can be generated by tumor cells or whether they may suppress immune cells, such as CD4, CD8 effector cells at tumor sites. The idiopathic CD4 lymphopenia (ICL) is a rare and heterogeneous syndrome defined by a reproducible reduction in the CD4 T-lymphocyte count $(<300$ cells $/ \mu \mathrm{L}$ or $<20 \%$ of total T cells) in the absence of HIV infection or other known causes of immunodeficiency (Smith et al., 1993).

\section{CD4 is critical for HIV entry}

In 1984, two seminal studies showed that CD4 is critical for HIV-1 entry. CD4 acts as primary receptor for human HIV-1 (PubMed: 2214026, PubMed: 16331979, PubMed: 9641677, PubMed: 12089508), primary receptor for HIV retroviruses and is down-regulated by HIV-1 Vpu (PubMed:17346169). A point mutation in CD4 (C868T) was found to be strongly correlated with increased susceptibility to HIV-1 infection in African populations (Oyugi et al., 2009). Recent reports have demonstrated that the CD4 protein is not only the cellular receptor for HIV-1 but also participates actively in post binding events important for infection and cell fusion. A significant association of SNPs in CD4 with HIV-2 infection has also been reported (Hennig et al., 2011). In HIV-1 infected cells, newly synthesized CD4 molecules are retained in the endoplasmic reticulum by a phenomenon termed receptor interference. This is the result of the formation of a complex between new CD4 and the viral gp160-env protein in the endoplasmic reticulum. The retained molecules of CD4 are degraded in this compartment by a mechanism induced by another HIV-1 protein, vpu.

Another viral protein interacting with CD4 is negative regulatory factor (nef). HIV-1-nef is a 206 amino acid, cytoplasmic protein that is nonessential for viral replication and associates with the plasma membrane through an $\mathrm{N}$-terminal myristic acid. It is responsible for the loss of CD4 from the cell surface by inducing its endocytosis and ultimately resulting in its lysosomal degradation. This phenomenon was shown to occur early in the course of the infection, before gp160-induced receptor interference. It is mediated by a dileucine motif similar to endocytosis and lysosomal degradation signals, contained in the membrane-proximal 20, residues of the CD4 cytoplasmic tail. In recent times, the CD4 molecule has become identified with more insidious events. The HIV virus has evolved to take advantage of CD4 and exploit it to invade CD4 T cells. The N-terminal extracellular domains of CD4 provide a perfect attachment site for the gp120 envelope glycoprotein of HIV, such that CD4 acts as part of a high affinity receptor complex that facilitates viral entry into CD4 cells. The destruction of the CD4 T cell population that follows HIV infection causes the overwhelming immune dysfunction suffered by AIDS patients.

\section{CD4 lymphocyte count and the risk of HIV-related diseases}

Some HIV-related diseases can be stratified to different CD4 lymphocyte count levels. Alan et al. (1998) summarize that HIV infected patients are susceptible to sinusitis, Kaposi's sarcoma, pneumonia and oral hairy leukoplakia, regardless of their CD4 count. If CD4 lymphocyte count level is below $200 / \mathrm{mm}^{3}$ i.e. in advanced HIV, toxoplasmosis, Pneumocystis carinii pneumonia, progressive multifocal leukoen- 
cephalopathy, molluscum contagiosum, Mycobacterium avium complex and bacillary angiomastosis all increase in incidence. In case of very advanced HIV disease, when CD4 counts are below $50 / \mathrm{mm}^{3}$, patients are at risk of central nervous system lymphoma, pseudomonas pneumonia, aspergillosis, cytomegalovirus retinitis and disseminated histoplasmosis. Marie et al. (2013) concluded that in virally suppressed HIV patients, an unexplained, major decline in CD4 count is associated with a markedly increased risk of cardiovascular disease, cancer and death.

\section{DNA methylation in CD4}

The DNA methylation level of the CD4 was strongly influenced by mastitis status in all comparisons and the results found by Wang et al. (2013) showed that mastitis could cause high methylation level of $C D 4$ in lactating cows. It regulates the expression of gene patterns in response to environmental cues including infections. An association of mastitis with the methylation patterns of $C D 4$ promoter in dairy cow peripheral blood cells was reported recently, suggesting that DNA methylation level of the CD4 promoter region can be used as a molecular marker for CM in dairy cows (Wang et al., 2013). DNA methylation in CD4 may play an important role in the susceptibility of mastitis and thus can be considered an advantageous epigenetic marker in mastitis resistant studies (Usman et al., 2016).

\section{Correlation CD4 with mastitis and production traits}

Researchers have shown that genetic markers associated with susceptibility to mastitis provide preliminary evidence for a phenotypic association between a single nucleotide polymorphism of ATP1A1, BoLADQA1, CARD15, CD4, CD18, CXCR2, $H M G B 1, M B L 1, T L R 1$ and TLR2 genes along with SCCs and clinical manifestations in dairy cows. Usman et al. (2018) found an association of novel polymorphisms in bovine $C D 4$ with SCC in CM cows. CD4 plays a vital role in the immune response to mastitis in dairy animals (Cao et al., 2012) and exerts a crucial role in the immune response of pathogen-induced mastitis in dairy cows (Zhao and Lacasse, 2015). An invasion of activated CD4+ T cells in the udder of cattle is a typical characteristic of mastitis (He et al., 2011). It plays an important role in the immune response to mastitis in dairy animals (Cao et al., 2012). Most importantly, CD4 protein and $\mathrm{CD} 4+\mathrm{T}$ cells play key roles in host resistance during the development of mastitis (Burton and Erskine, 2003; Rivas et al., 2007). Increased CD4+ T lymphocytes have been reported by many studies at the initial stages of mastitis (Taylor et al., 1997; Soltys and Quinn, 1999; Rivas et al., 2007). CD4 and $L A G-3$ could be potential candidate genes and the SNP2 in $L A G-3$ might be useful in CM markers integrated in Holstein cattle genomic selection (Usman et al., 2017). Both CD4 and $L A G$ 3 genes are located adjacent on chromosome (BTA) 5, which is within QTL for mastitis susceptibility (Meredith et al., 2013). He et al. (2011) reported that an influx of activated $C D 4+\mathrm{T}$ lymphocytes in the mammary gland is a distinct characteristic of mastitis. Some authors reported a significant effect of SNP in CD4 gene with SCS in Chinese Holstein cattle. Wang et al. (2013) found that the CpG Island in CD4 gene was highly methylated in mastitis cattle and was associated with lower gene expression than in healthy control. Recently an evidence for the role of 
CD4+ in protecting against mastitis is provided by the identification of SNPs in the bovine CD4 gene which is associated with SCC (Banos et al., 2013).

\section{Recommendations}

To control mastitis, enhance milk composition and increase milk yield, the following management measures should be adopted on priority basis.

- Rationalize feeding diets.

- Hygienic milking practices.

- Dipping teats immediately after milking with a safe and effective product (acetic acid or solution of vinegar).

- Regular udder screening.

- Pasteurization.

- Keeping accurate records of CM and SCCs in individual cow to assist in management.

- Selection against mastitis.

- Cross breeding between exotic and native species.

- CD4 should be considered in formulating breeding programs and marker assisted selection.

\section{Conclusion}

Genetics of mastitis resistance in dairy cattle has been studied for decades. SCC is a useful indicator of mastitis and is strongly correlated with CM. Therefore most studies focused on milk SCC or CM records as phenotypic measures to improve mastitis resistance. It is concluded that there is a negative correlation between milk production and mastitis resistance. Breeding strategies focusing only on milk production may lead to a population of cattle highly susceptible to mastitis which is undesirable. To avoid such conditions, mastitis resistance traits should be included in breeding strategies to produce animals that are genetically resistant to mastitis. Various molecular markers in cattle have proved to be beneficial for the dairy producers and breeders in improving milk production and various other performance traits by minimizing the incidence of economically important diseases such as mastitis. These molecular markers can be used efficiently in breeding and management decisions. CD4 gene plays an essential role in defense mechanism of mammary gland. The present study infers that $C D 4$ is a useful candidate gene and it possesses potentially essential molecular markers that can be used in breeding programs to improve the udder health and minimize the incidence of mastitis in dairy cattle using marker assisted selection.

\section{Conflict of interest}

There is no conflict of interest. 


\section{References}

A 1 a n C., Jung M.D., D o u g l a s S., P a a u w M.D. (1998). Diagnosing HIV-related disease using the CD4 Count as a guide. J. Gen. Intern. Med., 13:131-136.

A 1 hus si en M.N., D ang A.K. (2018). Milk somatic cells, factors influencing their release, future prospects, and practical utility in dairy animals: An overview. Vet. World, 11: 562-577.

A $1 \mathrm{maw}$ G., Molla W., Melaku A. (2012). Incidence rate of clinical bovine mastitis in selected smallholder dairy farms in Gondar Town in Ethiopia. Ethiop. Vet. J., 16: 93-99.

A $1 \mathrm{~m}$ e i d a R.A., C a l v in h o L.F., O li v e r S.P. (1998). Potential virulence factors of Streptococcus dysgalactiae associated with bovine mastitis. Vet. Micro., 61: 93-110.

A $\mathrm{m}$ e h J.A., Tari L.S. (2000). Observation on the prevalence of caprine mastitis in relation to predisposing factors in Maiduguri. Small Rumin. Res., 35: 1-5.

A te y a A.I., E 1 - S e a d y Y.Y., A tw a S.M., M e rgh an i B.H., S a y ed N.A. (2016). Novel single nucleotide polymorphisms in lactoferrin gene and their association with mastitis susceptibility in Holstein cattle. Genetika, 48: 199-210.

B a chay a H.A., R a za M.A., Murtaza S., A kb a r I.U.R. (2011). Subclinical bovine mastitis in Muzaffar Garh district of Punjab, Pakistan. J. Anim. Plant Sci., 21: 16-19.

Banos G., Wall E., Coffey M., Bagnall A., Gillespie S., Russe 11 G., Mc Nei1ly T. (2013). Identification of immune traits correlated with dairy cow health, reproduction and productivity. PLoS ONE, 8(6): 65766.

B i a le c ki E., M a ch o F.E., I va nov S., Paget C., Fon ta in e J. (2011). Spleen-resident CD4+ and CD4- CD8alpha- dendritic cell subsets differ in their ability to prime invariant natural killer T lymphocytes. PLoS One, 6: e26919.

B ila l M.Q., I qbal M.U., Muhammad G., Avais M., S aj id M.S. (2004). Factors affecting the prevalence of clinical mastitis in buffaloes around Faisalabad district. Pak. Intern. J. Agri. Bio., 6: $185-187$.

B o o n y a y a tra S., C h a is r i W. (2005). Incidence and prevalence of sub-clinical mastitis in smallholder dairy farms of Chiang Mai Province, Thailand. Chiang Mai Vet. J., 2: 25-30.

B o s cariol R., Pleas ance J., Piedrafita D.M., Ra adsma H.W., S pithill T.W. (2006). Identification of two allelic forms of ovine CD4 exhibiting a Ser183/Pro183 polymorphism in the coding sequence of domain 3. Vet. Immunol. Immunopathol., 113: 305-312.

Bradley A.J., Green M.J. (2001). Etiology of clinical mastitis in six Somerset dairy herds. Vet. Res., 148: 683-686.

Brodzki P., Kostro A., Brodzki W., Wawron J., Marczuk Kurek Ł. (2015). Inflammatory cytokines and acute-phase proteins concentrations in the peripheral blood and uterus of cows that developed endometritis during early postpartum. Theriogenology, 84: 11-18.

B urton J.L., Ers k in e R.J. (2003). Immunity and mastitis some new ideas for an old disease. Vet. Clin. Food Anim. J., 19: 1-45.

Burvenich C., Van M., Mehrzad J., Diez-Fraile A., Duchateau L. (2003). Severity of E. coli mastitis is mainly determined by cow factors. Vet. Res., 34: 521-564.

C a m p b e 11 J.R., M a r s h 11 R.T. (2016). Dairy Production and Processing: The Science of Milk and Milk Products: Waveland Press.

Cao D., Jing X., Wang X., Liu H., Chen D. (2012). Dynamics of CD4+ lymphocytes in mouse mammary gland challenged with Staphylococcus aureus. Asian J. Anim. Vet. Adv., 7: 1041-1048.

Coulon J.B., Gas qui P., B a rnou in J., Oli i er A., Pra de 1 P., P o m i è s D. (2002). Effect of mastitis and related germs on milk yield and composition during naturally-occurring udder infections in dairy cows. Anim. Res., 51: 383-393.

D e k kers J.C.M., Hos pital F. (2002). The use of molecular genetics in the improvement of agricultural populations. Nat. Rev. Gen., 3: 22-32.

D e ti 11 e u x J.C. (2002). Genetic factors affecting susceptibility of dairy cows to udder pathogens. Vet. Immunol. Immunopathol., 88: 103-110.

Die s e r S.A., Vis si o C., La sagno M.C., B ogni C.I., Larriestra A.J., O dierno L.M. (2014). Prevalence of pathogens causing subclinical mastitis in Argentinean dairy herds. Pak. Vet. J., 34: 124-126. 
F a r a m a r z N. (2008). Principles of Immunophenotyping. In: Hematopathology: Morphology, Immunophenotype, Cytogenetics and Molecular Approaches. Academic Press.

Fareed K.S., Khalid H.M., Allah B.K., Shajeela A., Muhammad I.B., Mehmoodu 1 - Has a n, Muhammad A., Tas e e A.K. (2015). Prevalence and economic losses of reproductive disorders and mastitis in buffaloes at Karachi, Pakistan. Indian J. Anim. Res., 389: 1-4.

F ourichon C., S e gers H., Malher X. (2000). Effect of disease on reproduction in the dairy cow: a meta-analysis. Theriogenology, 53: 1729-1759.

Gebreyohannes Y.T., Regas s a F.G., Kelay B. (2010). Milk yield and associated economic losses in quarters with subclinical mastitis due to Staphylococcus aureus in Ethiopian crossbred dairy cows. Trop. Anim. Health Prod., 42: 925-931.

G e r a S., Gu h a A. (2011). Assessment of acute phase proteins and nitric oxide as indicator of subclinical mastitis in Holstein $\times$ Haryana cattle. Ind. J. Anim. Sci., 81: 1029-1031.

G i 1 m o u r A., H a r v e y J. (1990). Society for Applied Bacteriology Symposium Series. 19:147S166S.

Glass E.J., Preston P.M., Springbett A., Craigmile S., Kirvar E., Wilkie G., B row n C.D. (2005). Bos taurus and Bos indicus (Sahiwal) calves respond differently to infection with Theileria annulata and produce markedly different levels of acute phase proteins. Int. J. Para., 35: 337-347.

G o d d a r d M.E., H a y e s B.J. (2009). Mapping genes for complex traits in domestic animals and their use in breeding programmes. Nat. Rev. Gen., 10: 381-391.

Gust a fs s on K., Germana S., S undt T.M., S a ch s D.H., Le Guern C. (1993). Extensive allelic polymorphism in the CDR2-like region of the miniature swine CD4 molecule. J. Immunol., 151: 1365-1370.

Hagnestam-Nielsen C., Osterga ard S. (2009). Economic impact of clinical mastitis in a dairy herd assessed by stochastic simulation using different methods to model yield losses. Animal, 3: 315-328.

Ha la sa T., Huijps K., O s teras O., Hogeve en H. (2007). Economic effects of bovine mastitis and mastitis management: A review. Vet. Quarterly, 29: 18-31.

H a m e e d K.G.A., S e n d e r G., K o rw i n - K o s s a k o w s k a A. (2007). Public health hazard due to mastitis in dairy cows. Anim. Sci. Pap. Rep., 25: 73-85.

He Y., Chu Q., Ma P., Wang Y., Zhang Q., Sun D., Zhang Y., Yu Y., Zhang Y. (2011). Association of bovine CD4 and STAT5b single nucleotide polymorphisms with somatic cell scores and milk production traits in Chinese Holsteins. J. Dairy Res., 78: 242-249.

Hennig B.J., Velez-Edwards D.R., Van Der Loeff M.F.S., B is seye C., Edwards T.L., T a c c o ne 11 i A., N o ve 11 i G., A a b y P., Ka y e S., S c ot t W.K., J a y e A. (2011). CD4 intragenic SNPs associate with HIV-2 plasma viral load and CD4 count in a community-based study from Guinea-Bissau, West Africa. J.A.I.D.S., 56: 1-8.

H ind s D.A., S tuve L.L., N ils en G.B., Halperin E., Eskin E., B allinger D.G., Fra-। z e r K.A., C ox D.R. (2005). Whole genome patterns of common DNA variation in three human populations. Science, 307: 1072-1079.

Hinrichs D., Stamer E., Junge W., Kalim E. (2005). Genetic analyses of mastitis data using animal threshold models and genetic correlation with production traits. J. Dairy Sci., 88: 2260-2268.

Hog e ve en H., Huijps K., Lam T.J.G.M. (2011). Economic aspects of mastitis: New developments. New Zealand Vet. J., 59: 16-23.

H o 1 m e s C.W., W i 1 s o n G.F. (1984). Milk production from pastures. Butterworths of New Zealand. Wellington, New Zealand.

H o r e j s i V. (2003). The roles of membrane microdomains (rafts) in T cell activation. Immunol. Rev., 191: 148-164.

International Dairy Federation (1999). Suggested interpretation of mastitis terminology. Bulletin of the International Dairy Federation, 338: 3-26.

Iraguha B., Hamudikuwanda H., Mushonga B., Kandiwa E., Mpatswenumugab o J.P. (2017). Comparison of cow-side diagnostic tests for subclinical mastitis of dairy cows in Musanze district, Rwanda. J.S.A.V.A., 88: 1464.

I s 1 a m M.A., I s 1 a m M.Z., R a h m a n M.S., I s 1 a m M.T. (2011). Prevalence of subclinical mastitis in dairy cows in selected areas of Bangladesh. Bangladesh J. Vet. Med., 9: 73-78. 
Joshi S., Gokhale S. (2006). Status of mastitis as an emerging disease in improved and periurban dairy farms in India. Ann. New York Acad. Sci., 1081: 74- 83.

K a r i m a G.A.H. (2013). Genetic basis of mastitis resistance in dairy cattle - a review. Ann. Anim. Sci., 13: 663-673.

Katsande S., Matope G., Ndengu M., P fukenyi D.M. (2013). Prevalence of mastitis in dairy cows from smallholder farms in Zimbabwe. J. Vet. Res., 80: E1-7.

Kehrli M.E., Shuster D.E. (1994). Factors affecting milk somatic cells and their role in health of the bovine mammary gland. J. Dairy Sci., 77: 619-627.

Ko ivula M., Mantysa ar i E.A., N eguss ie E., S erenius T. (2005). Genetic and phenotypic relationships among milk yield and somatic cell count before and after clinical mastitis. J. Dairy Sci., 88: 827-833.

Kolbehdari D., Wang Z., Grant J.R., Murdoch B., Prasad A., Xiu Z., Marques E., S t o th ard P., M o or e S.S. (2009). A whole genome scan to map QTL for milk production traits and somatic cell score in Canadian Holstein bulls. J. Anim. Breed. Genet., 126: 216-227.

Kono T., Koren a a H. (2013). Cytokine gene expression in CD4 positive cells of the Japanese pufferfish, Takifugu rubripes. PLoS ONE, 8(6): e66364.

Koskinen R., S a lomonsen J., Tregaskes C.A., Young J.R., Goodchild M., Bumste a d N. Vain i o O. (2002). The chicken CD4 gene has remained conserved in evolution. Immunogenetics, 54: 520-525.

Kozacinski L.M., Iladziosmanovi T., Majic I.K., Jole C.Z. (2002). Relationships between the results of mastitis tests, somatic cell counts and the detection of mastitis agents in milk. Paraxis Vet., 57: 255-260.

Leung R.K., Thomson K., Gallimore A., Jones E., Van den Broek M., Sierro S., Alsheikhly A.R., McMichael A., Rahemtulla A. (2001). Deletion of the CD4 silencer element supports a stochastic mechanism of thymocyte lineage commitment. Nat. Immunol., 2:1167-73.

Mak T.W., S a unders M.E. (2006). The immune response. 1st ed. Basic and Clinical Principals. Elsevier, Amsterdam.

Marie H., Gitte K., Carsten S., Larsen G.P., Court P., Niels O., Jan G. (2013). CD4 decline is associated with increased risk of cardiovascular disease, cancer, and death in virally suppressed patients with HIV. Clin. Infect. Dis., 57: 314-321.

Mattapallil J.J., Douek D.C., Hill B., Nishimura Y., Martin M., Roederer M. (2005). Massive infection and loss of memory CD4+ T cells in multiple tissues during acute SIV infection. Nature, 434: 1093-1097.

Megersa B., Chala T., A bunna F., Regass a A., B erhanu M., Etana D. (2010). Occurrence of mastitis and associated risk factors in lactating goats under pastoral management in Borana, Southern Ethiopia. Trop. Anim. Health. Prod., 42: 1249-1255.

M e ll e n b e r g e r R. (2001). California Mastitis Test (CMT): An invaluable tool for managing mastitis. Dept. of Animal Sciences, Michigan State University, pp. 1-9.

Memon M.I., M irbahar K.B., Memon M.R., A khtar N., Soomoro S.A., Dewani P. (1999). A study on the etiology of subclinical mastitis in buffaloes. Pakistan J. Agri. Eng. Vet. Sci., 15: $34-36$.

Meredith B., Lynn D., Berry D., Kearney F., Bradley D., Finlay E., Fahey A. (2013). A genome-wide association study for somatic cell score using the Illumina high-density bovine beadchip identifies several novel QTL potentially related to mastitis susceptibility. Front. Genet., 4: 229.

Miyagawa F., Nakamura Y., Miyashita K., Iioka H., Himuro Y., Ogawa K., Nishimura C., Nishikawa M., Mitsui Y., Ito Y., Ommori R. (2016). Preferential expression of CD134, an HHV-6 cellular receptor, on CD4 T cells in drug-induced hypersensitivity syndrome (DIHS)/drug reaction with eosinophilia and systemic symptoms (DRESS). J. Dermatol. Sci., 83: 151-154.

Moges N., Hailemariam T., Fentahun T., Chaine M., Melaku A. (2012). Bovine mastitis and associated risk factors in smallholder lactating dairy farms in Hawassa, Southern Ethiopia. Global Vet., 9: 441-446.

Muhas in A.V.N., Kumar A., Rahim A., Sebastian R., Mohan V., Dewangan P.P.M. 
(2014). An overview on single nucleotide polymorphism studies in mastitis research. Vet. World, 7: 416-421.

Mukherji B.I.J.A.Y., Guha A., Chakraborty N.G., Sivanandham M., Nashed A.L., S p orn J.R., Ergin M.T. (1989). Clonal analysis of cytotoxic and regulatory T cell responses against human melanoma. J. Exp. Med., 169: 1961-1976.

N a m H.M., K i m J.M., L i m S.K., J a n g K.C., J u n g S.C. (2010). Infectious aetiologies of mastitis on Korean dairy farms during 2008. J. R.V.S.C., 88: 372-374.

N de g w a E.N., Mule i C.M., M u n y n a S.J. (2000). The prevalence of subclinical mastitis in dairy goats in Kenya. J. South Afr. Vet. Assoc., 71: 25-27.

Oj o O.E., O y e kun le M.A., O gun le ye A.O., O tes ile E.B. (2009). Escherichi coli, O157:H7 in food animals in part of south-western Nigeria. Prevalence and in vitro antimicrobial susceptibility. Trop. Vet., 26: 23-30.

O liver S., G on zále z R., H o gan J., J a y a ra o B., O w e n s W. (2004). Microbiological procedures for the diagnosis of bovine udder infection and determination of milk quality, 4 th ed. National Mastitis Council, Verona, WI, USA, 1-28 pp.

Oviedo-Boyso J., Valdez-Alarcón J., Cajero-Juárez M., Ochoa-Zarzosa A., López-Meza J., Bravo-Patiño A., Baizabal-Aguirre V. (2007). Innate immune response of bovine mammary gland to pathogenic bacteria responsible for mastitis. J. Infect., 54: 399-409.

O y u gi J.O., Vouriot F.C., A li mont i J., Wayne S., Lu o M., A o Z., Ya o X., S e kaly R.P., Elli ot t L.J., S i m on s en J.N. (2009). A common CD4 gene variant is associated with an increased risk of HIV-1 infection in Kenyan female commercial sex workers. The J. Infect. Dis., 199: 1327-1334

P a n t S.D., S chenkel F.S., B a c a I.L., S harma B.S., K a r row N.A. (2007). Identification of single nucleotide polymorphisms in bovine CARD15 and their associations with health and production traits in Canadian Holsteins. BMC Genomics, 8: 421.

P ir za d a M., M a lh i K.K., K a m b o h A.A., R ind R., A bro S.H., L a kho S.A., B hut to K.R., Huda N. (2016). Prevalence of subclinical mastitis in dairy goats caused by bacterial species. J. Anim. Health Prod., 4: 55-59.

Riva s A.L., S c hw a ge r S.J., Gon zále z R.N., Qu i m b y F.W., A n d e r s on K.L. (2007). Multifactorial relationships between intramammary invasion by Staphylococcus aureus and bovine leukocyte markers. Can. J. Vet. Res., 71: 135.

Rollin E., Dhuyvetter K.C., Overton M.W. (2015). The cost of clinical mastitis in the first 30 days of lactation: An economic modeling tool. Prev. Vet. Med., 122: 257-64.

Roth s child M.F., Skow L., Lamont S.J. (2000). The major histocompatibility complex and its role in disease resistance and immune responsiveness. In: Breeding for disease resistance in farm animals, Axford R.F.E., Bishop S.C., Nicholas F.W., Owen J.B (eds.). CAB International, pp. 73-105

Rupp R., B o i chard D. (1999). Genetic parameters for clinical mastitis, somatic cell score, production, udder type traits, and milking ease in first lactation Holsteins. J. Dairy Sci., 82: 2198-2204

S a m m i u 11 a h M.U.D., S y e d M.A., K h a n M. (2000). Frequency and causes of culling and mortality in Holstein Friesian cattle in NWFP (Pakistan). J. Anim. Health. Prod., 20: 22-24.

Schroeder J. (2012). Bovine mastitis and milking management. North Dakota State University. https:/www.ag.ndsu.edu/pubs/ansci/dairy/as1129.pdf

Sh a r m a N., S in g h N.K., B h a d w a l M.S., (2011). Relationship of somatic cell count and mastitis: An overview. Asian Austral. J. Anim. Sci., 24: 429-438.

Shitandi A., Anakalo G., Galgalo T., Mwangi M. (2004). Prevalence of bovine mastitis amongst smallholder dairy herds in Kenya. Isr. J. Vet. Med., 59: 1-2.

S ing e r J.B. (2009). Candidate gene association analysis. In: Cardiovascular genomics. Humana Press, Totowa, NJ, pp. 223-230.

S m i th D.K., N e a l J.J., H o $1 \mathrm{~m}$ berg S.D. (1993). Unexplained opportunistic infections and CD4+ T-lymphocytopenia without HIV infection. An investigation of cases in the United States. The Centers for Disease Control Idiopathic CD4 T-lymphocytopenia Task Force. N. Engl. J. Med., 328: $373-379$. 
S mith S.J., Cas es S., Jens en D.R., Chen H.C., S ande E., Tow B., S an an D.A., Ra ber J., Eckel R.H., F are se Jr. R.V. (2000). Obesity resistance and multiple mechanisms of triglyceride synthesis in mice lacking Dgat. Nat. Genet., 25: 87.

S olt y s J., Q u in n M.T. (1999). Selective recruitment of T-cell subsets to the udder during staphylococcal and streptococcal mastitis: analysis of lymphocyte subsets and adhesion molecule expression. Infect. Immun., 67: 6293-6302.

S ong M., He Y., Zhou H., Z hang Y., Li X., Yu Y. (2016). Combined analysis of DNA methylome and transcriptome reveal novel candidate genes with susceptibility to bovine Staphylococcus aureus subclinical mastitis. Sci. Rep., 6: 29390.

S or e n s e n L.P., M ark T., M a d s e n P., L u nd M.S. (2009). Genetic correlations between pathogen specific mastitis and somatic cell count in Danish Holsteins. J. Dairy Sci., 92: 3457-3471.

S te a r M.J., B is sh op S.C., M a 11 a rd B.A., R a a d s m a H. (2001). The sustainability, feasibility and desirability of breeding livestock for disease resistance. Vet. Sci., 71: 1-7.

S w a n s on K.M., S te 1 w a g e $n$ K., D a v is S.R., Henders o n H.V., D a v is S.R., F a r r V.C., S in g h K. (2009). Transcriptome profiling of Streptococcus uberis-induced mastitis reveals fundamental differences between immune gene expression in the mammary gland and in a primary cell culture model. J. Dairy Sci., 92: 117-129.

Ta y 1 or B.C., K e e fe R.G., D e 11 ing e r J.D., N a k a mur a Y., Cull or J.S., S t o t t J.L. (1997). $\mathrm{T}$ cell populations and cytokine expression in milk derived from normal and bacteria-infected bovine mammary glands. Cell. Immunol., 182: 68-76.

Udd in M.N., Udd in M.B., A 1 - Mam un M., H a s s an M.M., Khan M.M.H. (2012). Small Scale dairy farming for livelihoods of rural farmers: constraint and prospect in Bangladesh. J. Anim. Sci. Adv., 2: 543-550.

U s m an T., Yu Y., Z ha i L., Li u C., Wang X., Wang Y. (2016). Association of CD4 SNPs with fat percentage of Holstein cattle. Genet. Mol. Res., 15, DOI: 10.4238/gmr.15038697.

U s m an T., Wang Y., S ong M., Wang X., D ong Y., Li u C., Wang S., Zhang Y., X i a o W., $\mathrm{Yu}$ Y. (2017). Novel polymorphisms in bovine CD4 and LAG-3 genes associated with somatic cell counts of clinical mastitis cows. Genet. Mol. Res., 17, DOI: 10.4238/gmr16039859.

U s m an T., Ya chun W., M in y an S., X i a W., Y i chun D., Cha o L., Shux i n g W., Yi Z., We i X., Y ing Y. (2018). Novel polymorphisms in bovine CD4 and LAG-3 genes associated with somatic cell counts of clinical mastitis cows. GMR, 17(1).

Viegher D.E.S., B arkema H.W., Stryhn. H., O p somer G., De Kruif A. (2005). Impact of early lactation somatic cell count in heifers on milk yield over the first lactation. J. Dairy Sci., 88: 938-947.

Wang Z., Hong J., S un W., Xu G., Li N., Chen X., Li u A., Xu L., S un B., Zhang J.Z. (2006). Role of IFN- $\gamma$ in induction of Foxp3 and conversion of CD4+ CD25-T cells to CD4+ Tregs. J. Clin. Invest., 116: 2434-2441.

Wang X.S., Zhang Y., He Y.H., Ma P.P., Fan L.J., Wang Y.C., Zhang Y.I., S un D.X., Z h a n g S.L., Wa n g C.D., S o n g J.Z. (2013). Aberrant promoter methylation of the CD4 gene in peripheral blood cells of mastitic dairy cows. Genet. Mol. Res., 12: 6228-6239.

W a n g K., Sh en T., S i e g a 1 G.P., We i S. (2017). The CD4/CD8 ratio of tumor-infiltrating lymphocytes at the tumor-host interface has prognostic value in triple-negative breast cancer. Human Pathol., 69: 110-117.

Winter P., Colditz I.G. (2002). Immunological responses of the lactating ovine udder following experimental challenge with Staphylococcus epidermidis. Vet. Immunol. Immunopathol., 89: 57-65.

Xu Y., Weatherall C., Bailey M., Alcantara S., De Rose R., Estaquier J., Wilson K., Suzuki K., Corbeil J., Cooper D.A., Kent S.J. (2013). Simian immunodeficiency virus infects follicular helper CD4 T cells in lymphoid tissues during pathogenic infection of pigtail macaques. J. Virol., 87: 3760-3773.

$\mathrm{Z}$ h a o X., L a c a s e P. (2018). Mammary tissue damage during bovine mastitis: Causes and control. J. Anim. Sci., 86: 57-65.

Zou Y.R., Sunshine M.J., Taniuchi I., Hatam F., Killeen N., Littman D.R. (2001). Epigenetic silencing of CD4 in T cells committed to the cytotoxic lineage. Nat. Genet., 29: 332-336.

Received: 20 VI 2019

Accepted: 18 II 2020 